\title{
Evidence-Based Surgical Guidelines for Treating Children with Wilms Tumor in Low-Resource Settings
}

\author{
Abdelhafeez Abdelhafeez ${ }^{1}$, Tea Reljic ${ }^{2}$, Ambuj Kumar ${ }^{2}$, Tahmina Banu ${ }^{3}$, Sharon Cox ${ }^{4}$, \\ Andrew Davidoff ${ }^{1}$, Ahmed Elgendy ${ }^{5}$, Khalil Ghandour ${ }^{6}$, Justin Gerstle ${ }^{7}$, Jonathan \\ Karpelowsky $^{8}$, Sue Kaste ${ }^{1}$, Nahla Kechiche ${ }^{9}$, Natia Esiashvili ${ }^{10}$, Abdulrasheed Nasir ${ }^{11}$, \\ Amon Ngongola ${ }^{12}$, Jin Marollano ${ }^{13}$, Amabelle Moreno ${ }^{14}$, Arlene Muzira ${ }^{15}$, Jeannette \\ Parkes $^{16}$, Lily Saldaña ${ }^{17}$, Jaime Shalkow-Klincovstein ${ }^{18}$, Gordan Vujanic ${ }^{19}$, Thelma \\ Velasquez $^{20}$, Kokila Lakoo ${ }^{21}$, Sheena Mukkada ${ }^{1}$, and Simone Abib ${ }^{22}$ \\ ${ }^{1}$ St. Jude Children's Research Hospital \\ ${ }^{2}$ University of South Florida College of Medicine \\ ${ }^{3}$ Chittagong Research Institute for Children Surgery (CRICS) \\ ${ }^{4}$ Division of Paediatric Surgery, Red Cross War Memorial Children's Hospital, Department \\ of Surgery, Faculty of Health Sciences, University of Cape Town, South Africa \\ ${ }^{5}$ Tanta University \\ ${ }^{6}$ King Hussein Cancer Center \\ ${ }^{7}$ Memorial Sloan Kettering Cancer Center \\ ${ }^{8} 12$ The Children's Hospital at Westmead, Division of Child \& Adolescent Health, The \\ University of Sydney, Children's Cancer Research Unit -Kids Research Institute \\ ${ }^{9}$ University Hospital Monastir, LR12SP13, University of Monastir \\ ${ }^{10}$ Emory University Winship Cancer Institute \\ ${ }^{11}$ University of Ilorin Teaching Hospital \\ ${ }^{12}$ University of Zambia University Teaching Hospital \\ ${ }^{13}$ Childhood Cancer International \\ ${ }^{14}$ University of the Philippines-Philippine General Hospital Department of Surgery \\ ${ }^{15}$ Uganda Cancer Institute \\ ${ }^{16}$ Groote Schuur Hospital \\ ${ }^{17}$ Instituto Nacional de Salud del Niño San Borja \\ ${ }^{18}$ National Institute of Paediatrics and ABC Cancer Centre \\ ${ }^{19}$ Sidra Medicine, Weill Cornell Medicine - Qatar \\ ${ }^{20}$ Unidad Nacional de Oncologia Pediatrica \\ ${ }^{21}$ Oxford University \\ ${ }^{22}$ Pediatric Oncology Institute - GRAACC - Federal University of São Paulo
}

February 18, 2022

\begin{abstract}
Survival of Wilms tumor (WT) is $>90 \%$ in high-resource settings but $<30 \%$ in low-resource settings. Adapting a standardized surgical approach to WT is challenging in low-resource settings, but a local control strategy is crucial to improving out-
\end{abstract}


comes. Objective: Provide resource-sensitive recommendations for the surgical management of WT. Methods: We performed a systematic review of PubMed and EMBASE through July 7, 2020, and used the GRADE approach to assess evidence and recommendations. Recommendations: Initiation of treatment should be expedited, and surgery should be done in a high-volume setting. Cross-sectional imaging should be done to optimize preoperative planning. For patients with typical clinical features of WT, biopsy should not be done before chemotherapy, and neoadjuvant chemotherapy should precede surgical resection. Also, resection should include a large transperitoneal laparotomy, adequate lymph node sampling, and documentation of staging findings. For WT with tumor thrombus in the inferior vena cava, neoadjuvant chemotherapy should be given before en bloc resection of the tumor and thrombus and evaluation for viable tumor thrombus. For those with bilateral WT, neoadjuvant chemotherapy should be given for 6-12 weeks. Neither routine use of complex hilar control techniques during nephron-sparing surgery, nor nephron-sparing resection for unilateral WT with a normal contralateral kidney is recommended. When indicated, postoperative radiotherapy should be administered within 14 days of surgery. Post-chemotherapy pulmonary oligometastasis should be resected when feasible, if local protocols allow omission of whole-lung irradiation in patients with non-anaplastic histology stage IV WT with pulmonary metastasis. Conclusion: We provide evidence-based recommendations for the surgical management of WT, considering the benefits/risks associated with limited-resource settings.

\section{INTRODUCTION}

Wilms tumor (WT), one of the most common solid tumors, is highly curable with affordable interventions. ${ }^{1}$ The majority $(90 \%)$ of patients with WT in high-income countries survive with chemotherapy, adequate surgical local control, and radiation therapy when indicated. However, survival in low-resource settings remains poor $(50 \%$ to $<30 \%)$, reflecting limitations in resources (physical and human) and a lack of process standardization. ${ }^{2,3}$ The World Health Organization's Global Initiative for Childhood Cancer targets WT as one of six index cancers included in attempts to reduce disparities in childhood cancer outcomes. ${ }^{4}$ Efforts to address resource limitations include workforce training and shared advocacy to establish sustainable resources required for multimodality therapy and family support. Although guidance from high-income countries is available, it may be difficult to implement in low- and middle-income countries (LMICs) due to differences in resources and health systems. For WT, a limited capacity to manage intraoperative bleeding and limited access to diagnostics and radiation therapy are key factors necessitating the adaptation of guidelines to address specific challenges in LMICs. The aim of this work is to provide resource-sensitive recommendations for the surgical management of pediatric WT in limited-resource settings.

\section{METHODS}

\section{Clinical practice guidelines}

The guidelines were developed following the GRADE (Grading of Recommendations, Assessment, Development, and Evaluation) method (Supplemental Table S1). ${ }^{5}$ The primary target audience for these guidelines is surgeons providing care to children with WT, particularly in settings with limited resources. The recommendations are also intended to be used by policymakers and senior managers as the basis for developing national and local WT protocols and policies and for supporting staff education and training.

\section{Composition of the guideline steering and development groups}

A Guideline Steering Group (GSD) was formed, consisting of two methodologists, two clinicians, and a research associate. The Guideline Development Group (GDG) members were identified through St. Jude Global, the International Society of Pediatric Surgical Oncology, and the Global Initiative for Children's Surgery. This group included content experts and a patient advocate and was constructed to maintain geographic and gender representation.

\section{Disclosure and management of potential conflicts of interest}

All members of the GSD and GDG provided conflict-of-interest disclosures prior to the voting process.

\section{Clinical questions}


The key questions addressed were formulated based on the assembled list of priority topics, questions, and critical outcomes from the scoping exercise identified by the GSG and GDG.

\section{Outcomes}

The GSD and GDG discussed and identified important outcomes for patients undergoing treatment for WT. The following outcomes were considered during the development of the recommendations: overall survival, mortality, tumor spillage, local recurrence, bleeding, complications, intensity of therapy, and wrong therapy.

\section{Summary of the evidence}

For questions with available data, evidence profiles were produced denoting the quality of evidence and summary of findings for each outcome. The GDG members were invited to review and comment on all evidence prior to the panel's meeting.

\section{Review of the evidence}

Selection criteria for each question were determined a priori . Only comparative studies (systematic reviews, randomized controlled trials, cohort studies, case-control studies, and cross-sectional studies) were considered for inclusion. If randomized data were available, data from nonrandomized studies were excluded. Electronic and manual searches were conducted for each question. We searched PubMed and EMBASE through July 7, 2020. Additionally, we manually reviewed the reference lists of all relevant systematic reviews and included studies to find additional eligible studies. The titles and abstracts of all identified references were reviewed by a clinician and a methodologist from the GSG. Studies identified for full-text review were then reviewed by all members of the GSG, and any reasons for exclusion were noted.

Data from included studies were extracted by two members of the GSG. The quality of evidence from each included study was assessed using the appropriate risk-of-bias tool for each study design. The Cochrane RCT tool was used for randomized controlled trials. ${ }^{6}$ The Newcastle-Ottawa tool was used for cohort and case-control studies. ${ }^{7}$ QUADAS was used for diagnostic accuracy studies. ${ }^{8}$ Data on outcomes from similar studies were pooled when appropriate by using the random-effects model. All analyses were performed using RevMan, ${ }^{9}$ and all information was summarized as evidence profile tables. ${ }^{10}$

\section{Development of recommendations}

All training, deliberations, and voting were conducted virtually. Prior to the panel's discussion, the GSG members presented the background and evidence profile for each key question. The GDG members discussed the benefits and disadvantages for patients, patients' preferences, clinical impact, and the feasibility of each proposed intervention.

\section{Grading recommendations}

All questions were converted into recommendations prior to anonymous voting. Panelists initially voted for or against each recommendation, followed by a vote on the strength of the recommendation (strong or weak). A simple majority of $>50 \%$ was considered in favor or against a recommendation. In the case of a tie, the text of the recommendation was modified to achieve a majority vote for or against the recommendation.

\section{External review}

The recommendations were peer-reviewed by an external review group, which provided overall feedback on the manuscript but did not change the voting outcomes.

\section{Update plan}

Guidelines are to be updated every 4 years.

\section{Source of funding}

This guideline development effort was supported by funds from the American Lebanese Syrian Associated Charities (ALSAC). 


\section{RESULTS}

The priority questions guiding the evidence review and synthesis for these guidelines are listed in (Supplemental Table S2). The glossary of terms and phrases and their meanings for the purposes of this guideline are summarized in Supplemental Table S3.

\section{RECOMMENDATIONS}

\section{Preoperative phase}

The panel recommends expedited initiation of treatment for the management of WT(Strong recommendation, Quality of evidence: Very Low).

Panel deliberation. WT is a rapidly growing malignancy with a doubling time of less than 2 weeks. Therefore, any delay in diagnosis or initiation of therapy (Supplemental Table S3) should be avoided. To facilitate early identification of patients with WT, community awareness should be raised regarding the signs and symptoms of WT, favorable outcomes with timely treatment, and the urgency to seek health care. Strengthening referral networks and prioritizing chemotherapy and surgery for childhood cancer are essential to facilitate access to timely care.

The panel suggests surgery at a high-volume setting for patients with WT undergoing resection. (Weak recommendation, Quality of evidence: Very Low; Table 1).

Panel deliberation. Cancer units may have the capacity to offer common diagnostic and treatment services; however, pediatric oncologic surgery requires multidisciplinary capacity available at national cancer referral centers. ${ }^{11-15}$ When scaling-up surgical oncology care (Supplemental Table S2), it is fundamental to balance the competing priorities of quality and access. National cancer centers should be accessible and well connected with a network of primary health and cancer units to deliver affordable, equitable, and high-quality care. The cost effectiveness of treating cancer in centers with higher capabilities was shown in the third edition of Disease Control Priorities. ${ }^{16}$

The panel recommends abdominal and pelvic cross-sectional imaging for preoperative planning in patients with suspected WT (Strong recommendation, Quality of evidence: Very Low).

Panel deliberation. Cross-sectional imaging provides more in-depth knowledge of tumor anatomy, including focality and extent, which is important for preoperative surgical planning. ${ }^{17-22}$ Surgeons should develop skills to adequately interpret cross-sectional imaging delineating tumor extent and laterality for surgical planning. Although there is a paucity of evidence to compare outcomes of ultrasound-guided management versus cross-sectional imaging-guided management, the latter approach provides more comprehensive images of the tumor and its critical anatomy for the operating surgeon. When cross-sectional imaging is not readily available, abdominal ultrasound imaging may be used to guide therapy and provide valuable details about tumor origin, extent, and laterality.

The panel suggests chest computerized tomography (CT)-guided staging for the management of WT. (Weak recommendation, Quality of evidence: Very Low; Table 2)

Panel deliberation. Chest CT is significantly more sensitive than chest x-ray; management guided by chest $\mathrm{CT}$ is associated with improved event-free survival but not overall survival. ${ }^{23-27}$ When CT is not available, chest x-ray may guide therapy without compromising overall survival.

The panel recommends against biopsy for patients with typical clinical features of WT. (Strong recommendation, Quality of evidence: Very Low)

Panel deliberation. Children who present with typical clinical features of WT (Supplemental Table S3), including age ( $>6$ months and $<7$ years), symptoms, laboratory test results, and imaging features, should receive neoadjuvant chemotherapy without tissue diagnosis. In the context of limited diagnostic capacity, routinely performing biopsies in patients with typical WT presentation may delay therapy and increase complications. Open biopsy can upstage WT, thereby compromising local control. ${ }^{28,29}$ 
The panel recommends biopsy (or upfront surgical resection when safe) for patients with renal tumor with an atypical clinical feature.(Strong recommendation, Quality of evidence: Very Low)

Panel deliberation. Other tumors that are not of renal origin, such as neuroblastoma and Burkitt lymphoma, should be excluded by clinical examination, laboratory investigations, and imaging. Patients who present with primary renal tumors but with clinical features that are atypical of WT should have tissue confirmation to plan therapy appropriately. Atypical presentations of WT include age older than 7 years or younger than 6 months, absence of imaging features that are typical of WT, infants with pulmonary metastases, extrapulmonary/hepatic metastases, lactate dehydrogenase levels more than four times the normal limits, and hypercalcemia (Supplemental Table S3). In these scenarios, pre-therapy biopsy is indicated. ${ }^{30}$ Image-guided core needle biopsy, if available, is the approach of choice; open biopsy is associated with the risk of tumor spillage and should be avoided. Alternatively, if the tumor is deemed resectable, then upfront resection may be considered to provide tissue diagnosis and plan appropriate further treatment.

\section{Operative phase}

The panel suggests neoadjuvant chemotherapy for the treatment of patients with the typical clinical features of WT. (Weak recommendation; Quality of evidence: Moderate; Table 3)

Panel deliberation. Neoadjuvant chemotherapy goals are to decrease tumor size, prevent tumor spillage, and risk-stratify postoperative treatment based on tumor response. Neoadjuvant chemotherapy is also associated with lowering the incidence of tumor spillage and decreasing the stage of the disease. ${ }^{31-33}$ This may be particularly relevant in limited-resource settings, where tumor stage at diagnosis is mostly advanced, and therapy intensification is challenging because of barriers to accessing radiation therapy and supportive care. In some settings, upfront surgical resection is considered a measure to adapt to issues related to chemotherapy supply chain and accessibility. In these cases, surgical resection is performed at the time of diagnosis at the reference center, followed by chemotherapy close to the patient's residence. Upfront resection may prevent the administration of inappropriate therapy in a small percentage of patients and may help select patients with very low-risk WT who can be treated with surgery alone. However, a surgery-only approach is more appropriate in the context of advanced diagnostic capacity supporting molecular and genomic tumor analysis. ${ }^{34,35}$

The panel suggests adequate trans-peritoneal laparotomy incision for resection of WT. (Weak recommendation; Quality of evidence: Very Low; Table 4)

Panel deliberation. WT surgical oncology principles include optimal visualization of vital anatomical structures to avoid injury, minimal tumor handling to prevent tumor spillage, and adequate surgical staging, particularly with lymph node sampling. Minimally invasive surgery is an attractive approach for treating small tumors; however, it should not compromise staging, lymph node sampling, or prevention of tumor capsule injury. $36-39$

The panel recommends adequate and documented surgical staging for the management of WT. (Strong recommendation; Quality of evidence: Very Low)

Panel deliberation. Postsurgical therapy relies on pathologic staging and surgical staging. In some scenarios, especially in the context of limited diagnostic capacity, documented surgical findings may be the only indicator of tumor spillage. Failure to perform lymph node sampling results in suboptimal tumor staging. This may lead to inadequate therapy or, conversely, to overtreatment with associated potential toxicities. Adequately documented surgical staging should include lymph node sampling and documentation of local invasion, seeding or tumor spillage, and vascular extension (Supplemental Table S3). ${ }^{28,40-45}$

The panel recommends sampling of lymph nodes at the time of resection of WT; pathologic assessment of lymph node histology before and after chemotherapy is feasible and of value in staging. (Strong recommendation; Quality of evidence: Very Low)

Panel deliberation. Determining lymph node involvement by histology is feasible both before and after 
neoadjuvant chemotherapy. ${ }^{46}$ Lymph node sampling is an integral part of staging and should be completed at the time of tumor resection, regardless of whether neoadjuvant chemotherapy was given. ${ }^{28,40-45}$ At the time of sampling, five to seven lymph nodes should be obtained to reduce the risk of a false-negative finding. ${ }^{41,44}$

The panel recommends neoadjuvant chemotherapy for the management of WT with inferior vena cava thrombus extension. (Strong recommendation; Quality of evidence: Very Low; Table 5)

Panel deliberation. Neoadjuvant chemotherapy is associated with the mitigation of complications during the surgical resection of WT with tumor thrombus in the inferior vena cava. Also, the need for cardiopulmonary bypass may be reduced in patients with pretreated supra-diaphragmatic vena cava thrombus. ${ }^{19,47-50}$ Neoadjuvant chemotherapy should be used in all patients with WT and tumor thrombus in the inferior vena cava; this is particularly relevant in limited-resource settings.

The panel recommends evaluating the presence of viable tumor cells within the thrombus for patients with WT and inferior vena cava extension. (Strong recommendation; Quality of evidence: Very Low)

Panel deliberation. Up to two thirds of tumor thrombi include viable tumor cells. ${ }^{51,52}$ Viable tumor thrombus in the context of incomplete macroscopic resection increases the risk of WT relapse and death. ${ }^{51,52}$ When complete gross resection of the tumor thrombus is achieved, the viability of the thrombus may be considered in determining the need for radiation therapy.

The panel recommends neoadjuvant chemotherapy followed by en bloc surgical resection of WT with the inferior vena cava thrombus extension. (Strong recommendation; Quality of evidence: Very Low)

Panel deliberation. Intravascular tumor extension increases the complexity of surgical resection and is associated with an increased risk of bleeding and other complications; however, resection is an integral part of the local control strategy of tumor thrombus. Complete resection of the tumor thrombus should be the goal of surgery whenever possible. ${ }^{51,52} \mathrm{WT}$ with inferior vena cava extension should be managed in a referral center. Extending neoadjuvant therapy beyond 6 weeks is not associated with improved surgical resection, further thrombus regression, or survival advantage. ${ }^{53}$

The panel recommends neoadjuvant chemotherapy for 6 to 12 weeks for patients with bilateral WT requiring nephron-sparing resection.(Strong recommendation; Quality of evidence: Very Low)

Panel deliberation. Tumor response to chemotherapy should be assessed at 6 weeks and 12 weeks; maximal tumor shrinkage occurs within the first 12 weeks of neoadjuvant chemotherapy. ${ }^{54}$ Tumors that show no response to chemotherapy after 6 weeks should be resected to prevent the side effects of protracted chemotherapy. Protracted chemotherapy may subject patients with stromal-type tumor to unnecessary toxicities or delay appropriate therapy for patients with anaplasia.

The panel suggests not to routinely use complex hilar techniques, including continuous vascular clamping and bench surgery, for bleeding control in patients with bilateral WT requiring nephron-sparing resection. (Weak recommendation; Quality of evidence: Very Low)

Panel deliberation. Large series have demonstrated that nephron-sparing surgery can be successfully completed using intermittent manual compression. ${ }^{55,56}$ However, selected patients may require more complex hilar control maneuvers. Surgeons should avoid prolonged ischemia (more than $20 \mathrm{~min})^{57,58}$ of the residual renal tissue, as it is associated with adverse outcome of renal function.

The panel suggests intraoperative histologic confirmation of uncertain margins in patients with WT requiring nephron-sparing resection.(Weak recommendation; Quality of evidence: Very Low)

Panel deliberation. Intraoperative margin biopsy should be considered for areas of uncertain margins and potential residual disease. Frozen sections, however, are not needed routinely, especially when the tumor was resected with either a margin of normal renal parenchyma or an intact capsule.

The panel suggests that nephron-sparing resection not be performed in patients with unilateral WT. (Weak recommendation; Quality of evidence: Very Low; Table 6) 
Panel deliberation. Nephron-sparing surgery for unilateral WT may be associated with increased risk of tumor spillage and is not the standard of care. ${ }^{59-63}$ Nephron-sparing surgery may be feasible, especially in small polar tumors; however, the primary priority is to achieve complete tumor resection with negative margins. Nephrectomy for unilateral WT is associated with high overall survival and high recurrence-free survival, with an extremely low incidence of renal failure in patients who do not have cancer-predisposition syndrome. Nephron-sparing surgery is indicated primarily for patients with unilateral WT in the context of cancer-predisposition syndrome or for those who have only one kidney.

\section{Postoperative phase}

The panel suggests postoperative abdominal radiation therapy within 14 days of surgery for patients with WT who require adjuvant radiation therapy. (Weak recommendation; Quality of evidence: Very Low)

Panel deliberation. Postoperatively, patients who need radiation therapy, especially those with high-risk WT, should be referred to a radiation oncologist within 1 week of surgery to receive abdominal radiation within 14 days. A radiation oncologist should be part of the multi-disciplinary team discussion of all patients at diagnosis, so referral and need for radiation therapy can be anticipated. Delayed radiation therapy of the abdominal primary tumor in non-metastatic patients is associated with suboptimal local control. ${ }^{64-66}$ In contrast, for patients with metastatic disease, radiation of the abdominal primary within 14 days post nephrectomy is of no clear significance. Therefore, in these patients, abdominal irradiation can be performed at the same time as lung irradiation (when indicated). Suboptimal nutritional status of patients should be considered when combining whole-lung radiation therapy with flank versus whole-abdomen radiation therapy.

The panel suggests that resection of residual pulmonary oligometastasis after completion of chemotherapy (when feasible) be considered in a setting of local protocols that allow the omission of whole-lung irradiation in patients with favorable histology, stage IV WT with pulmonary metastasis. (Weak recommendation; Quality of evidence: Very Low)

Panel deliberation. The decision to omit whole-lung irradiation is based on tumor biology, histological risk group of the abdominal primary, and chemosensitivity; and can be applied in the context of local treatment protocols. Lung irradiation can be avoided for patients with non-anaplastic histology who are complete responders (i.e., those who have no residual pulmonary metastases on a chest CT after chemotherapy). In patients with residual post-chemotherapy oligometastasis that are amenable to resection, radiation therapy can be omitted if there was no residual viable tumor in the surgically cleared nodules. ${ }^{60-62,67,68}$ Resection of residual nodules may be helpful when institutional protocols/guidelines allow the omission of whole-lung radiotherapy in patients with non-anaplastic histology and no viable tumor in respected lesions.

\section{DISCUSSION}

A clinician panel used the GRADE approach to produce evidence-based recommendations for the surgical management of pediatric WT. Nineteen recommendations were formulated to address preoperative, operative, and postoperative concerns identified by surgeons practicing in limited-resource settings. The guidelines address key questions prioritized by the panel in the three phases of patient care. The panel included multidisciplinary WT experts and maintained geographic and gender balance. It produced recommendations based on the available evidence and the identified priority outcomes and goals relevant in limited-resource settings.

A few themes were identified throughout panel deliberations. In a limited-resource setting, childhood tumor treatment is often effective only if the patient presents early; therefore, access to timely therapy is of paramount importance. In addition, a multidisciplinary childhood cancer team incorporating radiologists, pathologists, oncologists, radiation oncologists, and surgical specialists who discuss the case prior to any surgical intervention is essential to ensure high quality of care. Establishing a multidisciplinary team discussion or tumor boards is a cornerstone of building capacity for pediatric oncology care. One of the key limitations identified by the panel was a paucity of evidence to address the selected questions. When evidence exists, it 
is frequently of low quality. Well-designed, randomized controlled trials and cost-effectiveness analyses are needed to examine the impact of the included preoperative, operative, and postoperative interventions on the outcome of WT.

Diagnostic capacity is limited in LMICs, and this continues to pose a significant challenge to improving the outcomes of children with WT. When there is no access to cross-sectional images, ultrasound and x-ray images may guide therapy. The availability of pathologic analyses of frozen sections may also be limited in LMICs. However, there is no evidence to support the utility or cost-effectiveness of frozen sections in WT surgery. The therapeutic interventions recommended by the panel are affordable; most are considered standard of care across settings with various resources and are not associated with increased risk to patients. These recommendations are, therefore, practical and can serve as a reference for practice standardization that may improve the surgical outcomes of WT globally.

\section{Acknowledgement}

Thanks to Angela McArthur for editing the manuscript.

Thanks to Kathy Pritchard-Jones for providing helpful comments.

References

1. Leslie SW SP. Cancer, Wilms (nephroblastoma). Treasure Island (FL): StatPearls Publishing. 2018.

2. Ward ZJ, Yeh JM, Bhakta N, Frazier AL, Girardi F, Atun R. Global childhood cancer survival estimates and priority-setting: a simulation-based analysis. Lancet Oncol. 2019;20(7):972-983.

3. Chagaluka G, Paintsil V, Renner L, et al. Improvement of overall survival in the Collaborative Wilms Tumour Africa Project. Pediatr Blood Cancer. 2020;67(9):e28383.

4. Organization WH. WHO Global Initiative for Childhood Cancer: An overview. 2020.

5. Schünemann H BJ, Guyatt G, Oxman A, editors. GRADE handbook for grading quality of evidence and strength of recommendations. Updated October 2013. The GRADE Working Group. In:2013.

6. Higgins JPT SJ, Page MJ, Elbers RG, Sterne JAC. Chapter 8: Assessing risk of bias in a randomized trial. In: Higgins JPT TJ, Chandler J, Cumpston M, Li T, Page MJ, Welch VA (editors), ed. Cochrane Handbook for Systematic Reviews of Interventions version 6.2 (updated February 2021). Cochrane2021.

7. http://www.ohri.ca/programs/clinical_epidemiology/oxford.asp. Accessed.

8. Whiting P, Rutjes AW, Reitsma JB, Bossuyt PM, Kleijnen J. The development of QUADAS: a tool for the quality assessment of studies of diagnostic accuracy included in systematic reviews. BMC Med Res Methodol. 2003;3:25.

9. Review Manager (RevMan) [Computer program]. Version 5.4. The Cochrane Collaboration2020.

10. GRADEpro GDT: GRADEpro Guideline Development Tool [Software]. McMaster University, 2020 (developed by Evidence Prime, Inc.). Available from gradepro.org [computer program].

11. Richards MK, Goldin AB, Savinkina A, et al. The association between nephroblastoma-specific outcomes and high versus low volume treatment centers. J Pediatr Surg. 2017;52(1):104-108.

12. Gutierrez JC, Koniaris LG, Cheung MC, Byrne MM, Fischer AC, Sola JE. Cancer care in the pediatric surgical patient: a paradigm to abolish volume-outcome disparities in surgery. Surgery.2009;145(1):76-85.

13. Fuchs J, Kienecker K, Furtwangler R, et al. Surgical aspects in the treatment of patients with unilateral wilms tumor: a report from the SIOP 93-01/German Society of Pediatric Oncology and Hematology.Ann Surg. 2009;249(4):666-671.

14. Kramer S, Meadows AT, Pastore G, Jarrett P, Bruce D. Influence of place of treatment on diagnosis, treatment, and survival in three pediatric solid tumors. J Clin Oncol. 1984;2(8):917-923. 
15. Wijnen MH, Hulscher JB. Centralization of pediatric surgical care in the Netherlands: Lessons learned. J Pediatr Surg. 2021.

16. Gupta S, Howard SC, Hunger SP, et al. Treating Childhood Cancer in Low- and Middle-Income Countries. In: Gelband H, Jha P, Sankaranarayanan R, Horton S, eds. Cancer: Disease Control Priorities, Third Edition (Volume 3). Washington (DC)2015.

17. Khanna G, Rosen N, Anderson JR, et al. Evaluation of diagnostic performance of CT for detection of tumor thrombus in children with Wilms tumor: a report from the Children's Oncology Group. Pediatr Blood Cancer. 2012;58(4):551-555.

18. McDonald K, Duffy P, Chowdhury T, McHugh K. Added value of abdominal cross-sectional imaging (CT or MRI) in staging of Wilms' tumours. Clin Radiol. 2013;68(1):16-20.

19. Mushtaq I, Carachi R, Roy G, Azmy A. Childhood renal tumours with intravascular extension. $\mathrm{Br} J$ Urol. 1996;78(5):772-776.

20. Ritchey ML, Green DM, Breslow NB, Moksness J, Norkool P. Accuracy of current imaging modalities in the diagnosis of synchronous bilateral Wilms' tumor. A report from the National Wilms Tumor Study Group. Cancer. 1995;75(2):600-604.

21. Reiman TA, Siegel MJ, Shackelford GD. Wilms tumor in children: abdominal CT and US evaluation. Radiology. 1986;160(2):501-505.

22. Patel K, Mahboubi S, Sherman NH, Rosenberg HK. Diagnosis and follow-up of children with Wilms' tumor: correlative study of ultrasound and computed tomography. Report of eighteen cases. Ann Radiol (Paris). 1991;34(6-7):376-382.

23. Meisel JA, Guthrie KA, Breslow NE, Donaldson SS, Green DM. Significance and management of computed tomography detected pulmonary nodules: a report from the National Wilms Tumor Study Group. Int J Radiat Oncol Biol Phys. 1999;44(3):579-585.

24. Grundy PE, Green DM, Dirks AC, et al. Clinical significance of pulmonary nodules detected by CT and Not CXR in patients treated for favorable histology Wilms tumor on national Wilms tumor studies-4 and -5: a report from the Children's Oncology Group. Pediatr Blood Cancer. 2012;59(4):631-635.

25. Smets AM, van Tinteren H, Bergeron C, et al. The contribution of chest CT-scan at diagnosis in children with unilateral Wilms' tumour. Results of the SIOP 2001 study. Eur J Cancer.2012;48(7):1060-1065.

26. Wilimas JA, Kaste SC, Kauffman WM, et al. Use of chest computed tomography in the staging of pediatric Wilms' tumor: interobserver variability and prognostic significance. J Clin Oncol.1997;15(7):26312635 .

27. Wilimas JA, Douglass EC, Magill HL, Fitch S, Hustu HO. Significance of pulmonary computed tomography at diagnosis in Wilms' tumor. J Clin Oncol. 1988;6(7):1144-1146.

28. Shamberger RC, Guthrie KA, Ritchey ML, et al. Surgery-related factors and local recurrence of Wilms tumor in National Wilms Tumor Study 4. Ann Surg. 1999;229(2):292-297.

29. Irtan S, Van Tinteren H, Graf N, et al. Evaluation of needle biopsy as a potential risk factor for local recurrence of Wilms tumour in the SIOP WT 2001 trial. Eur J Cancer. 2019;116:13-20.

30. de la Monneraye Y, Michon J, Pacquement H, et al. Indications and results of diagnostic biopsy in pediatric renal tumors: A retrospective analysis of 317 patients with critical review of SIOP guidelines.Pediatr Blood Cancer. 2019;66(6):e27641.

31. D'Angio GJ, Evans AE, Breslow N, et al. The treatment of Wilms' tumor: Results of the national Wilms' tumor study. Cancer.1976;38(2):633-646. 
32. Lemerle J, Voute PA, Tournade MF, et al. Preoperative versus postoperative radiotherapy, single versus multiple courses of actinomycin D, in the treatment of Wilms' tumor. Preliminary results of a controlled clinical trial conducted by the International Society of Paediatric Oncology (S.I.O.P.). Cancer. 1976;38(2):647654 .

33. Mitchell C, Pritchard-Jones K, Shannon R, et al. Immediate nephrectomy versus preoperative chemotherapy in the management of non-metastatic Wilms' tumour: results of a randomised trial (UKW3) by the UK Children's Cancer Study Group. Eur J Cancer.2006;42(15):2554-2562.

34. Fernandez CV, Perlman EJ, Mullen EA, et al. Clinical Outcome and Biological Predictors of Relapse After Nephrectomy Only for Very Low-risk Wilms Tumor: A Report From Children's Oncology Group AREN0532.Ann Surg. 2017;265(4):835-840.

35. Shamberger RC, Anderson JR, Breslow NE, et al. Long-term outcomes for infants with very low risk Wilms tumor treated with surgery alone in National Wilms Tumor Study-5. Ann Surg. 2010;251(3):555-558.

36. Bouty A, Burnand K, Nightingale M, et al. What is the risk of local recurrence after laparoscopic transperitoneal radical nephrectomy in children with Wilms tumours? Analysis of a local series and review of the literature. J Pediatr Urol. 2018;14(4):327 e321-327 e327.

37. Ezekian B, Englum BR, Gulack BC, et al. Comparing oncologic outcomes after minimally invasive and open surgery for pediatric neuroblastoma and Wilms tumor. Pediatr Blood Cancer. 2018;65(1).

38. Duarte RJ, Cristofani LM, Denes FT, Filho VO, Tannuri U, Srougi M. Wilms tumor: a retrospective study of 32 patients using videolaparoscopic and open approaches. Urology.2014;84(1):191-195.

39. Romao RL, Weber B, Gerstle JT, et al. Comparison between laparoscopic and open radical nephrectomy for the treatment of primary renal tumors in children: single-center experience over a 5-year period.J Pediatr Urol. 2014;10(3):488-494.

40. Nanda RH, Shehata BM, Khoshnam N, et al. Impact of lymph node evaluation in adjuvant and neoadjuvant chemotherapy settings on survival outcomes in Wilms tumour: a review of 185 cases from a single institution. Pathology. 2017;49(1):19-23.

41. Saltzman AF, Smith DE, Gao D, et al. How many lymph nodes are enough? Assessing the adequacy of lymph node yield for staging in favorable histology wilms tumor. J Pediatr Surg.2019;54(11):2331-2335.

42. Fernandez CV, Mullen EA, Chi YY, et al. Outcome and Prognostic Factors in Stage III FavorableHistology Wilms Tumor: A Report From the Children's Oncology Group Study AREN0532. J Clin Oncol.2018;36(3):254-261.

43. Ehrlich PF, Anderson JR, Ritchey ML, et al. Clinicopathologic findings predictive of relapse in children with stage III favorable-histology Wilms tumor. J Clin Oncol.2013;31(9):1196-1201.

44. Kieran K, Anderson JR, Dome JS, et al. Lymph node involvement in Wilms tumor: results from National Wilms Tumor Studies 4 and 5. J Pediatr Surg. 2012;47(4):700-706.

45. Zhuge Y, Cheung MC, Yang R, Koniaris LG, Neville HL, Sola JE. Improved survival with lymph node sampling in Wilms tumor. J Surg Res. 2011;167(2):e199-203.

46. Vujanic GM, D'Hooghe E, Popov SD, Sebire NJ, Kelsey A. The effect of preoperative chemotherapy on histological subtyping and staging of Wilms tumors: The United Kingdom Children's Cancer Study Group (UKCCSG) Wilms tumor trial 3 (UKW3) experience. Pediatr Blood Cancer.2019;66(3):e27549.

47. Xu S, Sun N, Zhang WP, Song HC, Huang CR. Management of Wilms tumor with intravenous thrombus in children: a single center experience. World J Pediatr. 2019;15(5):476-482.

48. Cristofani LM, Duarte RJ, Almeida MT, Odone Filho V, Maksoud JG, Srougi M. Intracaval and intracardiac extension of Wilms' tumor. The influence of preoperative chemotherapy on surgical morbidity. Int 
Braz J Urol. 2007;33(5):683-689; discussion 689.

49. Lall A, Pritchard-Jones K, Walker J, et al. Wilms' tumor with intracaval thrombus in the UK Children's Cancer Study Group UKW3 trial.J Pediatr Surg. 2006;41(2):382-387.

50. Shamberger RC, Ritchey ML, Haase GM, et al. Intravascular extension of Wilms tumor. Ann Surg. 2001;234(1):116-121.

51. Boam TD, Gabriel M, Shukla R, Losty PD. Impact of neoadjuvant chemotherapy on thrombus viability in patients with Wilms tumour and caval extension: systematic review with meta-analysis. BJS Open.2021;5(3).

52. Dzhuma K, Powis M, Vujanic G, et al. Surgical management, staging, and outcomes of Wilms tumours with intravascular extension: Results of the IMPORT study. J Pediatr Surg. 2021.

53. Morris L, Squire R, Sznajder B, van Tinteren H, Godzinski J, Powis M. Optimal neoadjuvant chemotherapy duration in Wilms tumour with intravascular thrombus: A literature review and evidence from SIOP WT 2001 trial. Pediatr Blood Cancer. 2019;66(11):e27930.

54. Aydin B, Akyuz C, Yalcin B, et al. Bilateral Wilms tumors: Treatment results from a single center. Turk J Pediatr. 2019;61(1):44-51.

55. Spiegl HR, Murphy AJ, Yanishevski D, et al. Complications Following Nephron-Sparing Surgery for Wilms Tumor. J Pediatr Surg.2020;55(1):126-129.

56. Aldrink JH, Cost NG, McLeod DJ, et al. Technical Considerations for Nephron-Sparing Surgery in Children: What Is Needed to Preserve Renal Units? J Surg Res. 2018;232:614-620.

57. Thompson RH, Lane BR, Lohse CM, et al. Every minute counts when the renal hilum is clamped during partial nephrectomy. Eur Urol.2010;58(3):340-345.

58. Thompson RH, Lane BR, Lohse CM, et al. Comparison of warm ischemia versus no ischemia during partial nephrectomy on a solitary kidney.Eur Urol. 2010;58(3):331-336.

59. Cost NG, Sawicz-Birkowska K, Kajbafzadeh AM, et al. A comparison of renal function outcomes after nephron-sparing surgery and radical nephrectomy for nonsyndromic unilateral Wilms tumor. Urology.2014;83(6):1388-1393.

60. Szymik-Kantorowicz S, Urbanowicz W, Surmiak M, Sulislawski J. Therapeutic results in stage I Wilms' tumors in children - 15 years of surgical experience. Cent European J Urol. 2012;65(3):151-155.

61. Wilde JC, Aronson DC, Sznajder B, et al. Nephron sparing surgery (NSS) for unilateral wilms tumor (UWT): the SIOP 2001 experience.Pediatr Blood Cancer. 2014;61(12):2175-2179.

62. Zani A, Schiavetti A, Gambino M, Cozzi DA, Conforti A, Cozzi F. Long-term outcome of nephron sparing surgery and simple nephrectomy for unilateral localized Wilms tumor. J Urol. 2005;173(3):946-948; discussion 948 .

63. Haecker FM, von Schweinitz D, Harms D, Buerger D, Graf N. Partial nephrectomy for unilateral Wilms tumor: results of study SIOP 93-01/GPOH. J Urol. 2003;170(3):939-942; discussion 943-934.

64. Stokes CL, Stokes WA, Kalapurakal JA, et al. Timing of Radiation Therapy in Pediatric Wilms Tumor: A Report From the National Cancer Database. Int J Radiat Oncol Biol Phys. 2018;101(2):453-461.

65. Kalapurakal JA, Li SM, Breslow NE, et al. Influence of radiation therapy delay on abdominal tumor recurrence in patients with favorable histology Wilms' tumor treated on NWTS-3 and NWTS-4: a report from the National Wilms' Tumor Study Group. Int J Radiat Oncol Biol Phys.2003;57(2):495-499.

66. D'Angio GJ, Tefft M, Breslow N, Meyer JA. Radiation therapy of Wilms' tumor: results according to dose, field, post-operative timing and histology. Int J Radiat Oncol Biol Phys.1978;4(9-10):769-780. 
67. Pasqualini C, Furtwangler R, van Tinteren H, et al. Outcome of patients with stage IV high-risk Wilms tumour treated according to the SIOP2001 protocol: A report of the SIOP Renal Tumour Study Group.Eur J Cancer. 2020;128:38-46.

68. Dix DB, Seibel NL, Chi YY, et al. Treatment of Stage IV Favorable Histology Wilms Tumor With Lung Metastases: A Report From the Children's Oncology Group AREN0533 Study. J Clin Oncol.2018;36(16):1564-1570.

\section{Hosted file}

Table 1.docx available at https://authorea.com/users/461231/articles/556977-evidence-basedsurgical-guidelines-for-treating-children-with-wilms-tumor-in-low-resource-settings

\section{Hosted file}

Table 2.docx available at https://authorea.com/users/461231/articles/556977-evidence-basedsurgical-guidelines-for-treating-children-with-wilms-tumor-in-low-resource-settings

\section{Hosted file}

Table 3.docx available at https://authorea.com/users/461231/articles/556977-evidence-basedsurgical-guidelines-for-treating-children-with-wilms-tumor-in-low-resource-settings

\section{Hosted file}

Table 4.docx available at https://authorea.com/users/461231/articles/556977-evidence-basedsurgical-guidelines-for-treating-children-with-wilms-tumor-in-low-resource-settings

\section{Hosted file}

Table 5.docx available at https://authorea.com/users/461231/articles/556977-evidence-basedsurgical-guidelines-for-treating-children-with-wilms-tumor-in-low-resource-settings

\section{Hosted file}

Table 6.docx available at https://authorea.com/users/461231/articles/556977-evidence-basedsurgical-guidelines-for-treating-children-with-wilms-tumor-in-low-resource-settings 